Pacific Journal of Mathematics

REDUCING THE ORDER OF THE LAGRANGEAN FOR A 


\title{
REDUCING THE ORDER OF THE LAGRANGEAN FOR A CLASSICAL FIELD IN CURVED SPACE-TIME
}

\section{RICHARD ARENS}

\begin{abstract}
We show how the Lagrangean $L$ can be replaced by another, $L^{*}$, having the same extremals, but having only first order derivatives and being in fact a first degree polynomial in these derivatives.
\end{abstract}

1. Introduction. Whittaker [4] showed how to reduce the order and degree of a Lagrangean to 1 in the case of one-dimensional "space-time". As he points out, this leads instantly to Hamilton's canonical formalism. Rodrigues [3] showed how to do this without using coordinates in the configuration space. "Reducing the order" is not an adequate description of the construction since when the order is 1 (as it usually is) it still takes some work to make it of the first degree.

In [1] we treated the case of true (several dimensional) space-time. However, we took it to be $\mathbf{R}^{4}$, and we used coordinates in the field space.

Such a theorem is not usable when several coordinate systems must be used in space-time $M$. This is because the $L^{*}$ doing the desired things is not unique.

Our construction of $L^{*}$ here depends on the choice of an affine connection $\Gamma$ and a volume element $\omega$ in $M$. The result is not independent of the $\Gamma$ and $\omega$ chosen, but it is independent of coordinates.

2. $\pi$-manifolds. In this paper, a suitable order $N$ of differentiability is assumed.

Let $M$ be a manifold which we will call space-time. Let $P$ be another manifold. We will call it a $\pi$-manifold if there is defined on it a regular $\operatorname{map} \pi: P \rightarrow M$.

If $P$ and $Q$ are two $\pi$-manifolds, let $F(P, Q)$ be the class of all maps $f$ of open sets in $P$ into $Q$ for which

$$
\pi(f(p))=\pi(p)
$$

whenever $f(p)$ is defined. Let $p$ be a point of $P$ and let $f, g \in F(P, Q)$. Say $f \equiv g$ at $p$ if $f$ and $g$ agree up to the $N$ th order at $p$. Let $J^{N}(P, Q)$ be 
the set of equivalence classes. An element $C$ of $J^{N}(P, Q)$ may be represented by a pair $(p, f)$ where $C$ is an equivalence at $p$ and $f \in C$. Define $\pi(C)=\pi(p)=\pi(f(p))$. Then $J^{N}(P, Q)$ is also a $\pi$-space.

Let $X^{1}, \ldots, x^{m}, y^{1}, \ldots, y^{n}$ be coordinates in $P$ and $Q$ respectively. Then $\left(y^{i}\right)_{\lambda \ldots \nu}$ shall be the function defined in $J^{N}(P, Q)$ be saying that

$$
\left(y^{i}\right)_{\lambda \cdots \nu}(C)=\frac{\partial^{k}\left(y^{i} \circ f\right)}{\partial x^{\lambda} \cdots \partial x^{\nu}}(p) .
$$

Here $k$ must not exceed $N$.

A particularly useful kind of coordinates $x^{1}, \ldots, x^{m}$ are coordinates exponential at a point $p$. For this one must select an affine connection $\Gamma$ for $P$. Let $T^{1}(P)_{p}$ be the tangent space to $P$ at $p$. Select a linear coordinate system in $T^{1}(P) p$ and transfer these to $P$ using the exponential map defined by $\Gamma$ (see [2].)

3. Lagrangeans. In classical mechanics, a Lagrangean is a function defined on $\mathbf{R} \times T^{1}(Q)$, the latter being the tangent bundle of configuration space. Now $\mathbf{R} \times T^{1}(Q)$ is naturally isomorphic with $J^{1}(M, M \times Q)$ where $M=\mathbf{R}$, and $M \times Q$ has the projection on $M$. Since we want to use 2.1 we define Lagrangeans in this milieu.

Let $M$ be a manifold of dimension $m$. Let $S$ be a $\pi$-manifold. If coordinates $t^{1}, \ldots, t^{m}$ are chosen for $M$, then $t^{1} \circ \pi, \ldots, t^{m} \circ \pi$ are (independent, by the regularity of $\pi$ ) variables in $S$. We will abbreviate them to $t^{1}, \ldots, t^{m}$.

A Lagrangean (of order at most $N$ ) is an $m$-form $\Lambda$ defined on $J^{N}(M, S)$ such that in terms of coordinates $t^{1}, \ldots, t^{m}$ in $M$,

$$
\Lambda=L d t^{1} \wedge \cdots \wedge d t^{m}
$$

Let the class of these Lagrangeans be called $\mathscr{L}^{N}(S)$.

3.2. TheOREM. Let $M$ and $S$ be as above. Let $\Gamma$ be an affine connection for $M$. Let $\omega$ be a volume element for $M$. Let $K$ be the cotangent bundle of $J^{N}(M, S)$. Then $\Gamma$, $\omega$ define a mapping

$$
\mathscr{L}^{N}(S) \rightarrow \mathscr{L}^{1}(K), \quad \Lambda \rightarrow \Lambda^{*} .
$$

This mapping is linear and $1: 1$

3.4. $L^{*}$ is a polynomial of the first degree in the derivatives (necessarily only of the first order), and

3.5. $\Lambda^{*}$ has the same extremals as $\Lambda$.

The precise (and natural) meaning of 3.5 is given in $\$ 5$ below, together with the proof of 3.2 . 
Property 3.4 is useful for the following construction.

Let $x^{1}, \ldots, x^{n}$ (plus those $t^{1}, \ldots, t^{m}$ ) be coordinates in $S . \Lambda^{*}=L^{*} d t^{1}$ $\wedge \cdots \wedge d t^{m}$ and by $3.4, L^{*}$ is a sum of a term $-H$ depending only on the $x^{i}$ and $t^{\lambda}$, and a sum of terms like $a\left(x^{i}\right)_{\lambda}$. Thus $\Lambda^{*}$ is a sum of $-H d t^{1} \wedge \cdots \wedge d t^{m}$ and of terms like $a\left(x^{i}\right)_{\lambda} d t^{1} \wedge \cdots \wedge d t^{m}$. This latter term is congruent modulo $d x^{i}-\left(x^{i}\right)_{\lambda} d t^{\lambda}$ to $a d t^{1} \wedge \cdots \wedge d x^{i} \wedge \cdots d t^{m}$, $d x^{i}$ being in the $\lambda$ th place. Thus $\Lambda^{*}$ is congruent to an $m$-form involving only the $x^{i}$ and $t^{\lambda}$, that is, an $m$-form on $S$.

\section{A vector field $U_{\Gamma}$ on $J^{N}(M, S)$.}

4.1. ThEOREM. Let $\Gamma$ be an affine connection for $M$. Using $\Gamma$ one can construct a vector field $U_{\Gamma}$ on $J^{N}(M, S)$. Let $A \in J^{N}(M, S)$ and let $t^{1}, \ldots, t^{m}$ be exponential coordinates at $\pi(A)$ in $M$. Use $t^{1}, \ldots, t^{m}$ together with some further coordinates $x^{1}, \ldots, x^{n}$ as coordinates in $S$. Then at $A$,

$$
\begin{aligned}
U_{\Gamma}=\left(x^{i}\right)_{\lambda} \frac{\partial}{\partial\left(x^{i}\right)_{\lambda}}+2\left(x^{i}\right)_{\lambda \mu} \frac{\partial}{\partial\left(x^{i}\right)_{\lambda \mu}} & \\
& +\cdots+N\left(x^{i}\right)_{\lambda \cdots \nu} \frac{\partial}{\partial\left(x^{i}\right)_{\lambda \cdots \nu}}
\end{aligned}
$$

A sum is intended in 4.2. For example, by

$$
\left(x^{i}\right)_{\lambda \cdots \mu} \frac{\partial}{\partial\left(x^{i}\right)_{\lambda \cdots \mu}}
$$

where there are $k$ indices, we mean the sum over all sets of $k$ indices such that $1 \leq \lambda \leq \cdots \leq \mu \leq m$.

We now prove 4.1. Let $A=(a, f)$ be a point of $J^{N}(M, S)$. Express $f$ in terms of $t^{1}, \ldots, t^{m}$ :

$$
f=\varphi\left(t^{1}, \ldots, t^{m}\right)
$$

For sufficiently small real $s$ and $\left|t^{\lambda}\right|, f_{s}$ can be defined by

$$
f_{s}=\varphi\left(e^{s} t^{1}, \ldots, e^{s} t^{m}\right)
$$

Define the "moving" point $A_{s}$ in $J^{N}(M, S)$ as $\left(a, f_{s}\right)$. We define $U_{\Gamma}$ at $A$ to be the tangent to the curve $s \rightarrow A_{s}$ for $s=0$. 
We must show that its $\left(x^{i}\right)_{\lambda \ldots \mu}$ component is correctly represented in 4.2. Now

$$
\begin{aligned}
\left(x^{i}\right)_{\lambda \cdots \mu}\left(A_{s}\right) & =\left.\frac{\partial^{k}}{\partial t^{\lambda} \cdots \partial t^{\mu}}\left[x^{i} \circ f_{s}\right]\right|_{t=0} \\
& =\left.e^{k s} \frac{\partial^{k}}{\partial t^{\lambda} \cdots \partial t^{\mu}}\left[x^{i} \circ f\right]\right|_{t=0}=e^{k s}\left(x^{i}\right)_{\lambda \cdots \mu}(A) .
\end{aligned}
$$

Then we take $d / d s$ of this for $s=0$, obtaining

$$
k\left(x^{i}\right)_{\lambda \cdots \mu}
$$

as 4.2 asserts. The construction of $A_{s}$ is clearly independent of the coordinates.

4.3. Corollary. Let $K$ be the cotangent bundle $T_{1}(J)$ of $J=J^{N}(M, S)$. Then $\Gamma$ defines a real-valued function on $K$ whose expression in terms of coordinates is

$$
p_{i}^{\lambda} \overline{\left(x^{i}\right)_{\lambda}}+2 p_{i}^{\lambda \mu} \overline{\left(x^{i}\right)_{\lambda \mu}}+\cdots+N p_{i}^{\lambda \cdots \nu} \overline{\left(x^{i}\right)_{\lambda \cdots \nu}} .
$$

Here $p_{i}^{\lambda \cdots \mu}$ is the "momentum" coordinate dual to the configuration coordinate $\left(x^{i}\right)_{\lambda \ldots \mu}$ in $J$.

To prove 4.3 , we need only show that 4.4 is independent of the coordinate representation. A point $B$ is a pair $(A, g)$ where $A$ is a point of $J$ as before, and $g$ is a map from a neighborhood of $A$ into $\mathbf{R}$, where $g(A)=0$. The value of $p_{i}^{\lambda \cdots \mu}$ at $B$ is

$$
\left.\frac{\partial g}{\partial\left(x^{i}\right)_{\lambda \cdots \mu}}\right|_{A}
$$

The value of $\overline{\left(x^{i}\right)_{\lambda \cdots \mu}}$ at $B$ is just $\left(x^{i}\right)_{\lambda \cdots \mu}(A)$. Here the latter is defined in $J$, and the former is the coordinate defined in $T_{1}(J)$ as obtained from the latter and the projection $\rho$

$$
\begin{aligned}
& T_{1}(J)=K \\
& \quad \downarrow \\
& \quad J=J^{N}(M, S)
\end{aligned}
$$

Hence the value of 4.4 at $B$ is $U_{\Gamma}[g]$, the result of applying the operator $U_{\Gamma}$ to $g$, and is thus independent of coordinates. 
4.6. Corollary. Let $J$ and $K$ be as in 4.3. Let $P=J^{1}(M, K)$. Then $\Gamma$ defines a real-valued function on $P$ whose value at $C=(c, h)$ is

$$
\overline{p_{i}^{\lambda}} Z_{\lambda}^{i}+\overline{p_{i}^{\lambda \mu}} Z_{\lambda \mu}^{i}+\cdots+\overline{p_{i}^{\lambda \cdots \nu}} Z_{\lambda \cdots \nu}^{i}
$$

where $Z_{\lambda \cdots \mu}^{i}$ is the sum of all

$$
\left(\overline{\left(x^{i}\right)_{\lambda \cdots \hat{\sigma} \cdots \mu}}\right)_{\sigma} .
$$

Several explanations are needed.

4.81. In forming the sum $Z_{\lambda \ldots \mu}^{i}$ we select each index appearing in $\lambda \cdots \mu$, form 4.8 , and add the results. $\hat{\sigma}$ means delete ' $\sigma$ ' from the string $\lambda \cdots \mu$.

4.82. The $p_{i}^{\overline{\lambda \cdots \mu}}$ is $p_{i}^{\lambda \cdots \mu} \circ \xi$ where $\xi$ is the projection of $P=J^{1}(M, K)$ $\rightarrow K$. Hence, for an element $C=(c, h)$ of $P$, wherein $h$ maps a neighborhood of $c \in M$ into $K$,

$$
\overline{p_{l}^{\lambda \cdots \mu}}(C)=p_{l}^{\lambda \cdots \mu}(h(c))
$$

which is

$$
\left.\frac{\partial g}{\partial\left(x^{i}\right)_{\lambda \cdots \mu}}\right|_{A}
$$

if $h(c)=(A, g), A \in K$.

4.84. The bar on the $\left(x^{i}\right)_{\lambda \cdots \mu}$ means that (see 4.5)

$$
\overline{\left(x^{l}\right)_{\lambda \cdots \mu}}=\left(x^{i}\right)_{\lambda \cdots \mu} \circ \rho \text {. }
$$

For any real variable $z$ (and thus for $\overline{\left(x^{i}\right)_{\lambda \ldots}}$ ) on $K, 2.2$ with $N=1$ gives sense to $z_{\sigma}$ for $1 \leq \sigma \leq m$.

To begin the proof of 4.6 , we evaluate at $C=(c, h)$ of $P$. By 2.2 , the answer is

$$
\left.\frac{\partial}{\partial t^{\sigma}}\left[\overline{\left(x^{i}\right)_{\lambda \cdots \mu}} \circ h\right]\right|_{c}
$$

Let us write $\partial_{\sigma}$ for $\partial / \partial t^{\sigma}$. So

$$
Z_{\lambda \cdots \mu}^{i}=\sum \partial_{\sigma}\left[\left(x^{l}\right)_{\lambda \cdots \hat{\sigma} \cdots \mu} \circ \rho \circ h\right] .
$$

We need a somewhat more abstract version of 2.2. Let $\pi_{1}(C)=p$, $\pi_{2}(C)=h$ in 2.2. Replace $x^{\lambda}$ by $t^{\lambda}$ and $y^{i}$ by $x^{i}$, since that is the notation for the present instance. Then 2.2 says

$$
\left(x^{l}\right)_{\lambda \cdots \mu}=\left\{\partial_{\lambda} \cdots \partial_{\mu}\left(x^{i} \circ \pi_{2}\right)\right\} \circ \pi_{1} .
$$


Accordingly,

$$
Z_{\lambda \cdots \mu}^{i}=\left.\sum \partial_{\sigma}\left[\left\{\partial_{\lambda \cdots \hat{\sigma} \cdots \mu}\left(x^{i} \circ \pi_{2}\right)\right\} \circ \pi_{1} \circ \rho \circ h\right]\right|_{c} .
$$

We assert that $\pi_{1} \circ \rho \circ h$ is the identity map. First of all $\pi_{1}$ is the map $\pi$ for the $\pi$-space $J$. Then $\rho$ (4.5) is a $\pi$-space morphism, for that is the way in which $K$ is made into a $\pi$-space. So $\pi_{1} \circ \rho=\pi$. But $h$ has to satisfy 2.1, so $\pi \circ h=\pi$. But the $\pi$ for $M$ itself is the identity (on some neighborhood). Therefore

$$
Z_{\lambda \cdots \mu}^{i}=\sum \partial_{\sigma}\left\{\partial_{\lambda \cdots \hat{\sigma} \cdots \mu}\left(x^{i} \circ \pi_{2}\right)\right\}=k \partial_{\lambda \cdots \mu}\left(x^{i} \circ \pi_{2}\right)
$$

where $k$ is the length of the string $\lambda \cdots \mu$ with nothing deleted. So

$$
Z_{\lambda \cdots \mu}^{i}=\left.k\left\{\partial_{\lambda \cdots \mu}\left(x^{i} \circ \pi_{2}\right)\right\} \circ \pi_{1} \circ \rho \circ h\right|_{c}=k\left(x^{i}\right)_{\lambda \cdots \mu}(A)
$$

since $h(c)=(A, g)$. Combine this with 4.83 and obtain that this $Z$ term contributes

$$
k\left(x^{i}\right)_{\lambda \cdots \mu}(A) \frac{\partial g}{\partial\left(x^{i}\right)_{\lambda \cdots \mu}}
$$

to the sum 4.7. This sum is evidently $U_{\Gamma}[g]$ evaluated at $A$. Thus 4.7 is independent of the coordinates. Thus it defines the function for which 4.6 holds.

5. Proof of Theorem 3.2. Let $\Lambda$ be given. Choose coordinates in $M$. Then 3.1 defines $L$, a function defined on $J^{N}(M, S)$. We have projections

$$
\begin{gathered}
J^{1}(M, K)=P \\
\xi \downarrow \\
K=T_{1}(J) \\
\rho \downarrow \\
J=J^{N}(M, S) \\
\downarrow \\
S
\end{gathered}
$$

Hence $L$ defines a function on $J^{1}(M, K)$, which we denote by $L$ also, for simplicity. Using $\xi$, we can lift the function 4.4 up to a function $\varphi$ on $P$. The function defined by 4.6 may be called $\psi$.

Let

$$
\omega=\lambda d t^{1} \wedge \cdots \wedge d t^{m}
$$

$\lambda \neq 0$, be the volume element postulated in 3.2. 
We define

$$
L^{*}=L+\lambda(\psi-\varphi)=L-\lambda \varphi+\lambda \psi
$$

and

$$
\Lambda^{*}=L^{*} d t^{1} \wedge \cdots \wedge d t^{m}
$$

We consider assertion 3.4. 4.8 is a derivative of first order. The coefficients in 4.7 are mere coordinates, and the $Z$ 's are linear in the derivatives 4.8. Hence 3.4 holds.

To show 3.5 we mention first that $L^{*}$ has the form

$$
\begin{aligned}
L^{*}= & L+P_{l}^{\lambda}\left[\left(\overline{\left(X^{i}\right)}\right)_{\lambda}-\overline{\left(x^{i}\right)_{\lambda}}\right] \\
& +P_{i}^{\lambda \mu}\left[\left(\overline{\left(x^{i}\right)_{\lambda}}\right)_{\mu}+\left(\overline{\left(x^{i}\right)_{\mu}}\right)_{\lambda}-2 \overline{\left(x^{i}\right)_{\lambda \mu}}\right] \\
& \left.+P_{i}^{\lambda \mu \nu}\left[\left(\overline{\left(x^{i}\right)_{\mu \nu}}\right)_{\lambda}+\overline{\left(x^{i}\right)_{\lambda \nu}}\right)_{\mu}+\left(\overline{\left(x^{i}\right)_{\lambda \mu}}\right)_{\nu}-3 \overline{\left(x^{i}\right)_{\lambda \mu \nu}}\right]+\cdots,
\end{aligned}
$$

where $P_{i}^{\lambda}, P_{l}^{\lambda \mu}, \ldots$ are $\lambda$ (see 5.2) times the $p_{i}^{\lambda}, p_{l}^{\lambda \mu}, \ldots$ lifted up to $J^{1}(M, K)$ by the projections 5.1. It follows from [1, 3.4] that $L^{*}$ has the "same" extremals as $l$. The meaning of same is as follows. An extremal for $L^{*}$ gives us expressions

$$
\begin{aligned}
x^{l}= & f^{i}\left(t^{1}, \ldots, t^{m}\right), \\
\left(x^{i}\right)_{\lambda}= & u_{\lambda}^{i}\left(t^{1}, \ldots, t^{m}\right) \\
\left(x^{i}\right)_{\lambda \mu}= & u_{\lambda \mu}^{l}\left(t^{1}, \ldots, t^{m}\right) \\
& \vdots \\
P_{\lambda}^{l}= & g_{\lambda}^{i}\left(t^{1}, \ldots, t^{m}\right)
\end{aligned}
$$

If we abandon 5.4 and those following it, then 5.3 gives an extremal for $L$ in the usual sense. It is shown in [1] that

$$
u_{\lambda}^{l}=\frac{\partial f^{i}}{\partial t^{\lambda}}
$$

and so forth.

\section{Correction to [1].}

(a) Delete 6.5. (Prop. 6.6. remains true, with the $\varphi$ of 6.7.)

(b) Delete 6.9. (Better results are in the author's "The dynamic differential forms of the Klein-Gordon field and the conformal group", Jour. Geometry and Physics, Vol. 1, 1983.) 


\section{REFERENCES}

[1] Richard Arens, Reducing the order of a Lagrangean, Pacific J. Math., 93 (1981), 1-11.

[2] Sigurdur Helgason, Differential Geometry and Symmetry Spaces, Academic Press, 1962.

[3] P. R. Rodrigues, Sur les systèmes mécaniques generalizés, C. R. Acad. Sci. Paris 282, Ser A. (1976), 1307-1309.

[4] E. T. Whittaker, Analytic Dynamics..., Cambridge U. Press, 1937.

Received June 17, 1983.

UNIVERSITY OF CALIFORNIA

LOS ANGELES, CA 90024 


\title{
PACIFIC JOURNAL OF MATHEMATICS EDITORS
}

\author{
DONALD BABBITT (Managing Editor) \\ University of California \\ Los Angeles, CA 90024 \\ J. DugundJI \\ University of Southern California \\ Los Angeles, CA 90089-1113 \\ R. FINN \\ Stanford University \\ Stanford, CA 94305 \\ Hermann FlaschKa \\ University of Arizona \\ Tucson, AZ 85721
}

\author{
C. C. MOORE \\ University of California \\ Berkeley, CA 94720 \\ ARTHUR OGUS \\ University of California \\ Berkeley, CA 94720 \\ Hugo Rossi \\ University of Utah \\ Salt Lake City, UT 84112 \\ H. SAMELSON \\ Stanford University \\ Stanford, CA 94305
}

\begin{tabular}{|c|c|c|c|c|}
\hline \multicolumn{5}{|c|}{ ASSOCIATE EDITORS } \\
\hline R. ARENS & $\begin{array}{l}\text { E. F. BECKENBACH } \\
(1906-1982)\end{array}$ & NeumanN & F. WOLF & K. YosHIDA \\
\hline \multicolumn{5}{|c|}{ SUPPORTING INSTITUTIONS } \\
\hline \multirow{2}{*}{\multicolumn{2}{|c|}{$\begin{array}{l}\text { UNIVERSITY OF ARIZONA } \\
\text { UNIVERSITY OF BRITISH COLUMBIA }\end{array}$}} & \multicolumn{3}{|c|}{ UNIVERSITY OF OREGON } \\
\hline & & \multirow{2}{*}{\multicolumn{3}{|c|}{ UNIVERSITY OF SOUTHERN CALIFORNIA }} \\
\hline \multirow{2}{*}{\multicolumn{2}{|c|}{$\begin{array}{l}\text { CALIFORNIA INSTITUTE OF TECHNOLOGY } \\
\text { UNIVERSITY OF CALIFORNIA }\end{array}$}} & & & \\
\hline & & \multicolumn{3}{|c|}{$\begin{array}{l}\text { STANFORD UNIVERSITY } \\
\text { UNIVERSITY OF HAWAII }\end{array}$} \\
\hline & \multicolumn{3}{|c|}{ UNIVER SITY OF TOKYO } \\
\hline \multicolumn{2}{|c|}{$\begin{array}{l}\text { MONTANA STATE UNIVERSITY } \\
\text { UNIVERSITY OF NEVADA, RENO }\end{array}$} & \multicolumn{3}{|c|}{ UNIVERSITY OF UTAH } \\
\hline \multicolumn{2}{|c|}{ NEW MEXICO STATE UNIVERSITY } & \\
\hline \multicolumn{2}{|c|}{ OREGON STATE UNIVERSITY } & \multicolumn{3}{|c|}{$\begin{array}{l}\text { WASHINGTON STATE UNIVERSITY } \\
\text { UNIVERSITY OF WASHINGTON }\end{array}$} \\
\hline
\end{tabular}

The Supporting Institutions listed above contribute to the cost of publication of this Journal, but they are not owners or publishers and have no responsibility for its content or policies.

\begin{abstract}
Mathematical papers intended for publication in the Pacific Journal of Mathematics should be in typed form or offset-reproduced (not dittoed), double spaced with large margins. Please do not use built up fractions in the text of the manuscript. However, you may use them in the displayed equations. Underline Greek letters in red, German in green, and script in blue. The first paragraph must be capable of being used separately as a synopsis of the entire paper. In particular it should contain no bibliographic references. Please propose a heading for the odd numbered pages of less than 35 characters. Manuscripts, in triplicate, may be sent to any one of the editors. Please classify according to the scheme of Math. Reviews, Index to Vol. 39. Supply name and address of author to whom proofs should be sent. All other communications should be addressed to the managing editor, or Elaine Barth, University of California, Los Angeles, California 90024.

There are page-charges associated with articles appearing in the Pacific Journal of Mathematics. These charges are expected to be paid by the author's University, Government Agency or Company. If the author or authors do not have access to such Institutional support these charges are waived. Single authors will receive 50 free reprints; joint authors will receive a total of 100 free reprints. Additional copies may be obtained at cost in multiples of 50 .
\end{abstract}

The Pacific Journal of Mathematics is issued monthly as of January 1966. Regular subscription rate: $\$ 190.00$ a year (5 Vols., 10 issues). Special rate: $\$ 66.00$ a year to individual members of supporting institutions.

Subscriptions, orders for numbers issued in the last three calendar years, and changes of address should be sent to Pacific Journal of Mathematics, P.O. Box 969, Carmel Valley, CA 93924, U.S.A. Old back numbers obtainable from Kraus Periodicals Co., Route 100, Millwood, NY 10546.

The Pacific Journal of Mathematics at P.O. Box 969, Carmel Valley, CA 93924 (ISSN 0030-8730) publishes 5 volumes per year. Application to mail at Second-class postage rates is pending at Carmel Valley, California, and additional mailing offices. Postmaster: Send address changes to Pacific Journal of Mathematics, P.O. Box 969, Carmel Valley, CA 93924.

\section{PUBLISHED BY PACIFIC JOURNAL OF MATHEMATICS, A NON-PROFIT CORPORATION}




\section{Pacific Journal of Mathematics}

\section{Vol. 116, No. 2 December, 1985}

Richard Arens, Reducing the order of the Lagrangean for a classical field in curved space-time ................................ 209

Shiferaw Berhanu, W. Wistar (William) Comfort and James Dolan Reid, Counting subgroups and topological group topologies $\ldots \ldots \ldots \ldots \ldots 217$

Michael James Cambern, Isomorphisms of spaces of norm-continuous

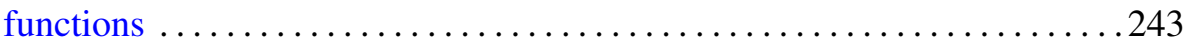

Gerald Arthur Edgar and Jun Feng Zhao, The ordering structure on

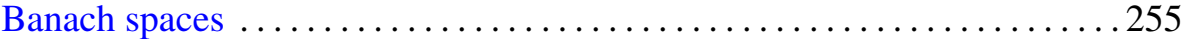

Irving Leonard Glicksberg, An analogue of Liapounoff's convexity theorem for Birnbaum-Orlicz spaces and the extreme points of their unit

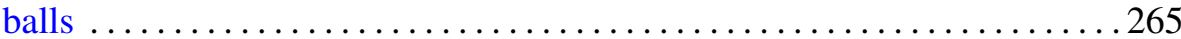

Daniel Heifetz, $p$-adic oscillatory integrals and wave front sets . ........285

José E. Pantoja, Liftings of supercuspidal representations of $\mathrm{Gl}_{2} \ldots \ldots \ldots 307$

Steven C. Pinault, An isoperimetric inequality for surfaces stationary with respect to an elliptic integrand and with at most three boundary

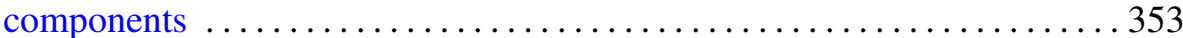

Georges Pinczon, Nonlinear multipliers and applications .............. 359 